\title{
Tracking Solid State Dynamics in Spray-Dried Protein Powders at Infrared and Terahertz Frequencies
}

\author{
Talia A. Shmool ${ }^{\mathrm{a}, 1}$, Maarten Batens ${ }^{\mathrm{c}, 1}$, Jan Massant ${ }^{\mathrm{b}}$, \\ Guy Van den Mooter ${ }^{\mathrm{c}}$, J. Axel Zeitler ${ }^{\mathrm{a}, *}$ \\ ${ }^{a}$ Department of Chemical Engineering and Biotechnology, University of Cambridge, \\ Cambridge, United Kingdom \\ ${ }^{b}$ Biological Formulation Development, UCB Pharma, Braine l'Alleud, Belgium \\ ${ }^{c}$ Drug Delivery and Disposition, KU Leuven, Leuven, Belgium
}

\begin{abstract}
Keywords: Spray-Dried Protein Powders, Bovine Serum Albumin, Terahertz, Infrared, Dynamics
\end{abstract}

\section{Introduction}

Therapeutic protein powders can be prepared by spray-drying. This process is known to result in solid particles of relatively narrow size distribution and high yield and purity [1,2]. Additionally, the spray-drying process is rapid, semi-continuous, cost-effective, reproducible and scalable. The process transforms a liquid into dry particles by atomising the liquid feed in a

Abbreviations: BSA: Bovine serum albumin; THz-TDS: Terahertz time-domain spectroscopy; FTIR: Fourier-transform infrared spectroscopy; mDSC: Modulated differential scanning calorimetry; PES: Potential energy surface; VDOS: Vibrational density of states; SSE: Sum of squared errors; RMSE: Root mean square error; SD:

Standard deviation

${ }^{*}$ Corresponding author

Email address: jaz22@cam.ac.uk (J. Axel Zeitler)

${ }^{1}$ Contributed equally to this work 
hot drying gas stream [3]. One of the main advantages of spray-drying is that a wide range of formulations, including heat-sensitive materials, can be dried using this technique since the droplet surface will retain the wet-bulb temperature rather than the temperature of the hot drying gas, provided evaporation is taking place at the droplet surface. By the time the evaporation at the droplet/particle surface stops, the drying gas will already have cooled down, thus limiting the heat exposure of the formulation components to the relatively high inlet gas temperatures, and, in combination with the short process duration, making spray-drying a feasible process for heat-sensitive materials, including proteins [1, 2, 3]. While spray-drying is a well established process for small molecules, the additional challenge of ensuring protein stability of the dried product during storage currently limits its use for biopharmaceutical products [2, 4]. A major concern during the spray-drying process is the entire or partial unfolding of proteins due to their high susceptibility to migrate to the air-liquid interfaces where the surface energies can cause the protein to expose hydrophobic regions, resulting in facilitated protein-protein interactions and ultimately aggregation [5]. In order to prevent such undesired aggregation non-ionic surfactants, for example polysorbate, are often used to prevent accumulation of protein at the air-liquid interface, as these small and more mobile surfactants will preferentially position themselves at the interfaces [6]. To put more generally, the excipients of a formulation are vital in providing stability to the protein by maintaining its native conformation during the spray-drying process.

When determining the chemical stability of a formulation molecular mobility is a key factor to consider, as an increase in molecular mobility has 
been directly linked to an increase in chemical degradation of a material, and therefore its storage stability [7]. In order to gain a deeper understanding of the effects of excipients and changes in protein structure it is insightful to understand the changes in the molecular mobility of a material and its structural dynamics. Terahertz time-domain spectroscopy (THz-TDS) is a relatively recent technique which can be used to investigate the vibrational dynamics of a material [8]. The advantage of this method is that it is a non-contact technique that can be used to measure molecular mobility and relaxation dynamics of amorphous materials over a broad temperature range at frequencies of $0.1-3 \mathrm{THz}$. At least two dielectric relaxation processes, the primary, or $\alpha$-relaxation process and the secondary, or $\beta$-relaxation process, are commonly observed in amorphous materials, including those containing peptides and proteins. The $\alpha$-relaxation process can be observed at temperatures above the calorimetric glass transition temperature $\left(T_{\mathrm{g}}\right)$ and we therefore designated this glass transition temperature as $T_{\mathrm{g}, \alpha}$. The relaxation process associated with the Johari-Goldstein $(\mathrm{JG}) \beta$-relaxation can still be observed at temperatures below $T_{\mathrm{g}, \alpha}$. At $T_{\mathrm{g}, \beta}$, the secondary glass transition, its relaxation time exceeds 100 seconds and the JG $\beta$ process can no longer be observed experimentally $[9,10,11,12$, 13]. For macromolecular systems, the $\alpha$-relaxation process is considered to represent large-scale mobility, whereas the secondary or $\beta$-relaxation processes have been associated with local, small-scale mobility. Similar to the $\alpha$-relaxation, the JG $\beta$-relaxation is a universal property of disordered materials and the two processes have been linked by means of a coupling model [14]. We have previously demonstrated that THz-TDS can be used to detect $T_{\mathrm{g}, \alpha}$ and $T_{\mathrm{g}, \beta}[15,16]$. The exact origin 
and molecular mechanisms associated with the $\alpha$ - and $\beta$-relaxation processes are still subject to some debate. Specifically the JG $\beta$-relaxation, is considered to be predominantly associated with the intermolecular degrees of freedom of a material $[17,18]$. It is worth noting that the potential energy surface (PES) model proposed by Goldstein is the most intuitive and comprehensive model to understand the molecular dynamics in amorphous systems, and that intra- and intermolecular processes are always fundamentally coupled by means of the PES [19]. Furthermore, it is also important to highlight that there are non-JG, very low energy barrier, secondary relaxation processes, such as those due to the methyl rotations, that will still take place at temperatures below $T_{\mathrm{g}, \beta}$.

The aim of this work is to understand the molecular mobility behaviour, the flexibility and thermally induced conformational changes of three distinct spray-dried formulations. Specifically, we investigate the effect of different commonly used excipients, including trehalose, L-arginine $\mathrm{HCl}$, and polysorbate 20 on three distinct formulations with bovine serum albumin (BSA) as the model protein. We propose a relationship between the relaxation dynamics and the molecular structure of the spray-dried protein formulations.

\section{Experimental Methods}

\subsection{Materials}

Lyophilised bovine serum albumin (BSA), $\mathrm{D}(+)$-trehalose dihydrate, Larginine $\mathrm{HCl}$, and polysorbate 20 were obtained from Sigma-Aldrich (Steinheim, Germany) and used as received. 
Table 1: Composition of aqueous feed solutions for spray-drying.

\begin{tabular}{llc}
\hline Formulation & Component & $\begin{array}{c}\text { Concentration } \\
\mathrm{mg} \mathrm{ml}^{-1}\end{array}$ \\
\hline F1 & BSA & 100.00 \\
& Trehalose & 34.23 \\
F2 & BSA & 100.00 \\
& Trehalose & 34.23 \\
F3 & L-arginine $\mathrm{HCl}$ & 50.56 \\
& BSA & 100.00 \\
& Trehalose & 34.23 \\
& L-arginine $\mathrm{HCl}$ & 50.56 \\
& Polysorbate 20 & 0.49 \\
\hline
\end{tabular}

\subsection{Spray-Dried Sample Preparation}

\subsubsection{Preparation of Feed Solutions}

Feed solutions for spray-drying were prepared by dissolving all the formulation components in ultrapure water (Type $1, \rho \geq 18.2 \mathrm{M} \Omega \mathrm{cm}$ at $25^{\circ} \mathrm{C}$ ) in a beaker up to approximately $75 \%$ of the final volume of $25 \mathrm{~mL}$. Once clear solutions were obtained, the solutions were transferred to $25 \mathrm{~mL}$ volumetric flasks and diluted further to their final volumes. An overview of the feed solutions for spray-drying is given in Table 1 .

\subsubsection{Spray-Drying}

The feed solutions were subsequently spray-dried using a a ProCepT Micro-Spray (ProCepT nv, Zelzate, Belgium) equipped with the Bi-fluid Mi- 
Table 2: Spray-drying process parameters.

\begin{tabular}{ll}
\hline Process Parameter & Setpoint \\
\hline Inlet air flow rate & $400 \mathrm{~L} \mathrm{~min}^{-1}$ \\
Inlet air temperature & $423 \mathrm{~K}$ \\
Nozzle air flow & $12 \mathrm{~L} \mathrm{~min}^{-1}$ \\
Feed rate & $3 \mathrm{~mL} \mathrm{~min}^{-1}$ \\
\hline
\end{tabular}

cro nozzle with $0.4 \mathrm{~mm}$ nozzle tip, using compressed air for atomisation, and a single cylindrical process column connected to the glass cone with filtered air (high efficiency particulate air) used as drying gas. Due to the limited batch sizes $(25 \mathrm{~mL})$, the medium cyclone was used in combination with the small collection vessel. An overview of the spray-drying process parameters and theoretical resultant powder compositions, corrected for the measured residual water content, are given in Table 2 and Table 3, respectively. Following the spray-drying process, samples were collected under nitrogen atmosphere and directly transferred from the collection vessel into Type I, clear, tubular glass injection vials (Schott AG, Mainz, Germany), which were purged with nitrogen gas before being sealed with FluroTec ${ }^{\circledR}$ rubber injection stoppers (West Pharmaceutical Services, West Whiteland Township, PA, USA) and aluminium crimp seals (Adelphi Healthcare Packaging, West Sussex, UK). Additionally, excipient mixtures, i.e. solutions with the same composition as the feed solutions described in Table 1 with the omission of BSA, were dried using the same spray-drying process. Calculated powder compositions, corrected for the measured residual water content, are also given in Table 4. 
Table 3: Calculated composition of spray-dried powders, and lyophilised excipient mixture corrected for measured residual water.

\begin{tabular}{llc}
\hline Formulation & Component & Concentration \\
& & $\% \mathrm{~m} / \mathrm{m}$ \\
\hline F1 & BSA & 71.66 \\
& Trehalose & 24.53 \\
& Residual water & 3.81 \\
F2 & BSA & 52.53 \\
& Trehalose & 17.98 \\
& L-arginine HCl & 26.56 \\
& Residual water & 2.93 \\
F3 & BSA & 52.43 \\
& Trehalose & 17.95 \\
& L-arginine HCl & 26.51 \\
& Polysorbate 20 & 0.26 \\
& Residual water & 2.85 \\
\hline
\end{tabular}




\subsubsection{Lyophilisation}

In addition to the spray-dried formulations, excipient mixtures were lyophilised using an Alpha 2-4 LSCplus freeze-dryer (Martin Christ Gefriertrocknungsanlagen GmbH, Osterode am Harz, Germany). Prior to lyophilisation, feed solutions were filtered using a $0.2 \mu \mathrm{m}$ filter and $4 \mathrm{ml}$ of the feed solution were dispensed per $10 \mathrm{ml}$ clear glass vial. Subsequently the vials were submerged in liquid nitrogen to flash freeze the solutions and reduce the risk of crystallisation during cooling. The vials were then placed into the freeze-dryer, which was pre-cooled to a shelf temperature of $238.15 \mathrm{~K}$, subjected to the lyophilisation programme described in Table 5 and then stored at $268.15 \mathrm{~K}$ pending characterisation. Calculated powder compositions, corrected for the measured residual water content, are given in Table 4. Amorphicity for all freeze- and spray-dried samples was confirmed by subjecting aliquots of the lyophilised powders to X-ray powder diffraction analysis (data not shown) using an X'pert PRO X-ray diffractometer (PANalytical, Almelo, The Netherlands) using the method described in more detail by Batens et al. [20] and by means of modulated differential scanning calorimetry.

\subsection{Modulated Differential Scanning Calorimetry (mDSC)}

A Q2000 Differential Scanning Calorimeter (TA Instruments, New Castle, DE, USA) was used to determine the calorimetric glass transition temperature $\left(T_{\mathrm{g}, \mathrm{mDSC}}\right.$, defined by the onset temperature) for each material. $2-3 \mathrm{mg}$ of sample material were placed in hermetically sealed aluminium pans under a constant flow nitrogen atmosphere (flow rate $50 \mathrm{ml} \mathrm{min}^{-1}$ ) and cooled at a rate of $3 \mathrm{~K} \mathrm{~min}^{-1}$ from room temperature to approximately $243 \mathrm{~K}$. The samples were subsequently heated at a rate of $10 \mathrm{~K} \mathrm{~min}^{-1}$ to $295 \mathrm{~K}$ and then at 
Table 4: Calculated composition of dried excipient mixtures, corrected for measured residual water.

\begin{tabular}{|c|c|c|c|}
\hline & & $\begin{array}{c}\text { Spray-dried } \\
\text { excipient }\end{array}$ & $\begin{array}{c}\text { Lyophilised } \\
\text { excipient }\end{array}$ \\
\hline \multirow[t]{2}{*}{ Formulation } & Component & mixture & mixture \\
\hline & & $\% \mathrm{~m} / \mathrm{m}$ & $\% \mathrm{~m} / \mathrm{m}$ \\
\hline \multirow[t]{2}{*}{ F1_Exc } & Trehalose & 94.36 & 97.93 \\
\hline & Residual water & 5.64 & 2.07 \\
\hline \multirow[t]{3}{*}{ F2_Exc } & Trehalose & 38.86 & 39.17 \\
\hline & L-arginine $\mathrm{HCl}$ & 57.39 & 57.86 \\
\hline & Residual water & 3.75 & 2.97 \\
\hline \multirow[t]{4}{*}{ F3_Exc } & Trehalose & 39.15 & 38.52 \\
\hline & L-arginine $\mathrm{HCl}$ & 57.83 & 56.89 \\
\hline & Polysorbate 20 & 0.56 & 0.55 \\
\hline & Residual water & 2.45 & 4.04 \\
\hline
\end{tabular}


Table 5: Lyophilisation parameters used for excipient mixtures.

\begin{tabular}{lcccc}
\hline & Section & Duration & $\begin{array}{c}\text { Shelf } \\
\text { temperature } \\
\text { K }\end{array}$ & $\begin{array}{c}\text { Vacuum } \\
\text { Pa }\end{array}$ \\
\hline Loading & 1 & & 238.15 & \\
\hline Freezing & 2 & 2 hours & 238.15 & \\
\hline & 3 & 10 minutes & 238.15 & 10.0 \\
& 4 & 5 hours & 253.15 & 10.0 \\
& 5 & 5 hours & 253.15 & 10.0 \\
& 6 & 5 hours & 258.15 & 10.0 \\
& 7 & 5 hours & 258.15 & 10.0 \\
& 8 & 5 hours & 263.15 & 10.0 \\
& 9 & 5 hours & 273.15 & 10.0 \\
& 10 & 10 hours & 283.15 & 10.0 \\
& 11 & 5 hours & 293.15 & 10.0 \\
& 12 & 5 hours & 293.15 & 10.0 \\
\hline \multirow{4}{*}{ Final drying } & 13 & 10 minutes & 293.15 & 0.1 \\
& 14 & 2 hours & 298.15 & 0.1 \\
& 15 & 12 hours & 298.15 & 0.1 \\
\hline
\end{tabular}


$5 \mathrm{~K} \mathrm{~min}^{-1}$ to $373 \mathrm{~K}$ with a modulation amplitude of $0.50 \mathrm{~K}$ and a period of 80 seconds.

Standard aluminium pans (TA Instruments) were used for measuring the dried excipient mixtures (Table 4) as higher glass transition temperatures were expected for these formulations and the application of a higher maximum temperature, i.e. $423.15 \mathrm{~K}$, could cause a pressure build-up inside hermetic pans due to evaporation of the residual water that was present in the dried excipient mixtures. Samples were heated from $243.15 \mathrm{~K}$ to $423.15 \mathrm{~K}$ at a rate of $5 \mathrm{~K} \mathrm{~min}^{-1}$ with a modulation amplitude of $0.24 \mathrm{~K}$ and period of 40 seconds, after an isothermal period of 5.00 minutes while the cell was continuously purged with dry nitrogen at $50 \mathrm{~mL} \mathrm{~min}^{-1}$. $T_{\mathrm{g}, \mathrm{mDSC}}$ determination was performed in the same way as for the BSA-containing formulations, by using the onset temperature.

For samples where the $T_{\mathrm{g}, \mathrm{mDSC}}$ could not be determined directly from the measured thermograms, or where measurements could not be repeated due to sample restrictions (as was the case for formulation F1, see Table 3), the glass transition temperatures were estimated using the Fox equation (Equation 1), where $w_{i}$ and $T_{\mathrm{g}, i}$ are the weight fraction and the glass transition temperature of the pure excipient.

$$
\frac{1}{T_{\mathrm{g}, \text { mix }}}=\sum_{1}^{i} \frac{w_{i}}{T_{\mathrm{g}, i}}
$$

The Fox equation offers a relatively simple method to estimate glass transition temperatures, but caution should always be taken when interpreting the results, as it assumes equal densities of, and equal hetero- and homonuclear intermolecular interactions occurring between, the formulation compo- 
nents. Estimates made using the Fox equation are therefore prone to a higher level of uncertainty than those made using the Gordon-Taylor equation, but the latter could not be applied due to difficulties with determining the densities of the pure amorphous phases. Using the glass transition temperature for amorphous water of $136 \mathrm{~K}$ as reported in the literature [21, 22], and the $T_{\mathrm{g}, \mathrm{mDSC}}$ that was determined for the dried excipient mixtures, the glass transition temperature of the anhydrous excipient mixture, $T_{\mathrm{g}, \mathrm{ex}}$, was estimated as follows:

$$
T_{\mathrm{g}, \mathrm{ex}}=\frac{w_{\mathrm{ex}}}{\frac{1}{T_{\mathrm{g}, \mathrm{mDSC}}}-\frac{w_{\mathrm{H}_{2} \mathrm{O}}}{T_{\mathrm{g}, \mathrm{H}_{2} \mathrm{O}}}}
$$

The estimated glass transition temperature of the formulation was then obtained by using the value of $T_{\mathrm{g}, \mathrm{BSA}}=407.15 \mathrm{~K}$ for the glass transition temperature of neat BSA as reported by Mizuno and Pikal [23].

$$
\frac{1}{T_{\mathrm{g}, \mathrm{Fox}}}=\frac{w_{\mathrm{ex}}}{T_{\mathrm{g}, \mathrm{ex}}}+\frac{w_{\mathrm{H}_{2} \mathrm{O}}}{T_{\mathrm{g}, \mathrm{H}_{2} \mathrm{O}}}+\frac{w_{\mathrm{BSA}}}{T_{\mathrm{g}, \mathrm{BSA}}}
$$

Depending on whether the spray- or freeze-dried excipient mixtures were used in the calculation the transition temperatures were referred to as $T_{\mathrm{g}, \text { Fox }, \mathrm{SD}}$ or $T_{\mathrm{g}, \text { Fox }, \mathrm{FD}}$ respectively.

\subsection{Karl Fischer Titration}

A $831 \mathrm{KF}$ Coulometer with generator electrode, coupled to a 774 Oven Sample Processor (Metrohm AG, Herisau, Switzerland) were used for water content determination in the spray-dried and lyophilised powders according to the method described by Batens et al. [20]. Protein containing samples 
were measured in triplicate, where the residual water contents calculated for the excipient mixtures were based on unique measurements.

\section{5. $T H z-T D S$}

\subsubsection{Sample Preparation}

All sample vials were opened and subsequent sample preparation was carried out in a glove bag (Sigma-Aldrich AtmosBag) which was continuously purged with dry nitrogen gas (relative humidity $<1 \%$ ) to avoid moisture sorption from atmospheric water vapour. The spray-dried powder samples were pressed into $13 \mathrm{~mm}$ diameter flat-faced pellets, at a load of 0.5 metric tons. The produced pellets were each between $300-700$ microns in thickness, and were placed between two z-cut quartz windows. This sandwich structure was immediately sealed in the sample holder following preparation.

\subsubsection{Experimental Setup and Data Analysis}

The THz-TDS spectra were acquired using the methodology introduced by Shmool and Zeitler [24]. The absorption coefficient and the refractive index of each sample were calculated using a modified method for extracting the optical constants from terahertz measurements based on the concept introduced by Duvillaret et al. $[25,26]$. The changes in dynamics of the samples were analysed by investigating the change in the absorption coefficient at a frequency of $1 \mathrm{THz}$ as a function of temperature using the methodology introduced previously $[24,8]$.

\subsection{Fourier Transform Infrared Spectroscopy (FTIR)}

A TENSOR II FTIR Spectrometer (Bruker, Billerica, MA, USA) equipped with a Golden Gate attenuated total reflectance (ATR) accessory (Specac, 
Orpington, UK) was used to obtain FTIR spectra of spray-dried BSA formulations in situ while they were heated from 303.15 K to 393.15 K. Spectra were recorded (64 scans) following a 5 minute isothermal temperature equilibration period, at each $10 \mathrm{~K}$ step. Both reference and sample spectra were recorded with a resolution of $2 \mathrm{~cm}^{-1}$ over a range of 4000 to $850 \mathrm{~cm}^{-1}$. Reference spectra were acquired at each $10 \mathrm{~K}$ temperature step and automatically subtracted from sample spectra by the included OPUS software package (Bruker). For the spray- and freeze-dried excipient mixtures, additional spectra for comparison were collected at ambient temperature using a Vertex 70 Fourier transform infrared (FTIR) spectrometer (Bruker) at 64 scans per spectrum, which included the 4000 to $400 \mathrm{~cm}^{-1}$ range at a spectral resolution of $2 \mathrm{~cm}^{-1}$, followed by the automatic subtraction of a reference spectrum, also acquired at ambient temperature and using the same spectral settings.

Data pre-processing and processing was performed using version 7.5 of the OPUS software package (Bruker). Data pre-processing was performed on the absorbance spectra and consisted of atmospheric compensation $\left(\mathrm{CO}_{2}\right.$ and $\mathrm{H}_{2} \mathrm{O}$ ), rubber band baseline correction (128 points, with exclusion of $\mathrm{CO}_{2}$ bands) and vector normalisation, all of which were performed over the entire spectral range. Next, peaks were identified over the entire spectral range using the proprietary peak picking function of the OPUS software. The peak picking method used had a sensitivity of $1 \%$ and was based on the second derivative obtained using the Savitzky-Golay algorithm with 9 smoothing points. Only the peaks in the amide I $\left(1700-1600 \mathrm{~cm}^{-1}\right)$ and amide II (1580 - $\left.1520 \mathrm{~cm}^{-1}\right)$ bands were taken into consideration for further 
evaluation [27]. If the standard deviation of the averaged wavenumber value for a peak, i.e. the averaged peak value across the measured temperatures, exceeded $2 \mathrm{~cm}^{-1}$, cf. the spectral resolution, the outlier was removed from the average and regarded as a separate peak after which the standard deviation was calculated and evaluated again.

\section{Results and Discussion}

\subsection{Thermal Analysis}

Table 6 provides an overview of the $T_{\mathrm{g}, \mathrm{mDSC}}$ data determined using the thermograms (see supporting information) that were obtained for the lyophilised and spray-dried excipient mixtures (see Table 4). The data show a decrease in $T_{\mathrm{g}, \mathrm{mDSC}}$ following $T_{\mathrm{g}, \mathrm{mDSC}, \mathrm{F} 1 \_E x c}>T_{\mathrm{g}, \mathrm{MDSC}, \mathrm{F} 2{ }_{-} \mathrm{Exc}}=T_{\mathrm{g}, \mathrm{mDSC}, \mathrm{F} 3 \_ \text {Exc }}$ and $T_{\mathrm{g}, \mathrm{mDSC}, \mathrm{F} 1 \_ \text {Exc }}>T_{\mathrm{g}, \mathrm{mDSC}, \mathrm{F} 2 \text { Exx }}>T_{\mathrm{g}, \mathrm{mDSC}, \mathrm{F} 3 \text { _Exc }}$ for the spray-dried and lyophilised excipient mixtures, respectively. Based on the data collected for the lyophilised and spray-dried excipient mixtures alone, we cannot discriminate whether this effect is caused by the presence of the additional excipients or the variation in residual water content (see Table 4), since both could contribute to the plasticising effect on the trehalose matrix. However, when taking into the account the calculated anhydrous glass transition temperatures summarised in Table 7, it becomes apparent that not only water but also BSA and the other excipients in the trehalose matrix contribute to the plasticising effect of the sample. This happens regardless of whether the spray-dried or lyophilised excipient mixtures were used in the calculations of the estimated glass transition. It should be mentioned that the calculated

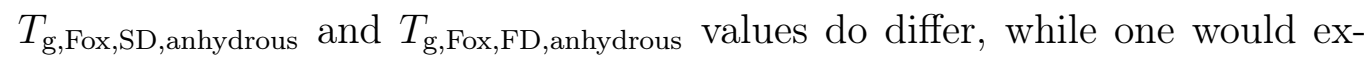


pect these to be identical, but as these are estimates based on experimental values, a certain level of discrepancy could not be avoided. Notwithstanding the discrepancies, the observations and conclusions regarding the plasticising effects of L-arginine $\mathrm{HCl}$ and polysorbate 20 remain valid.

Table 6: $T_{\mathrm{g}, \mathrm{mDSC}}$ of dried excipient mixtures.

\begin{tabular}{lcccc}
\hline Formulation & Spray-dried excipient mixture & Lyophilised excipient mixture \\
& $n=3$ & $\mathrm{~K}$ & & $\mathrm{~K}$ \\
& & $\pm \mathrm{SD}$ & 315.18 & $\pm \mathrm{SD}$ \\
\hline F1_Exc & 331.57 & 4.49 & 293.26 & 2.23 \\
F2_Exc & 312.65 & 0.59 & 288.98 & 2.00 \\
F3_Exc & 312.78 & 1.56 & & 2.67 \\
\hline
\end{tabular}

\subsection{Terahertz Spectroscopy}

The terahertz spectra of all formulations showed an increase in absorption with frequency and temperature over the entire investigated range, and no discrete spectral features were present for the three spray-dried formulations, in line with previous measurements of amorphous molecular solids. As expected, each spectrum was dominated by the monotonous increase with frequency, characteristic for the rising flank of the peak due to the vibrational density of states (VDOS) [15]. To further investigate the relationship between the increase of absorption coefficient and temperature we examined the temperature dependent changes in absorption losses at a frequency $1 \mathrm{THz}$ in more detail. 
Table 7: Estimated glass transition temperatures of anhydrous samples, $T_{\mathrm{g}, \text { Fox }}$, calcu-

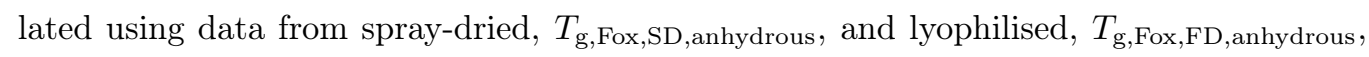
excipient mixtures using Equation 1.

\begin{tabular}{lcc}
\hline Formulation & $T_{\mathrm{g}, \text { Fox }, \text { SD,anhydrous }}$ & $T_{\mathrm{g}, \text { Fox,FD,anhydrous }}$ \\
\hline F1 & 362.69 & 324.19 \\
F2 & 329.28 & 304.00 \\
F3 & 323.32 & 303.31 \\
F1_Exc & 410.44 & 397.35 \\
F2_Exc & 378.38 & 362.94 \\
F3_Exc & 374.41 & 362.04 \\
\hline
\end{tabular}

The changes in absorption at a frequency of $1 \mathrm{THz}$ with temperature for the formulations are plotted in Figure 1. Figure 1d shows the behaviour of the spray-dried excipient mixture of trehalose, arginine, and polysorbate 20 used for F3 but the absence of BSA. For all of the formulations, the change in absorption with temperature can be observed to take place over three distinct regions and two transition temperatures, $T_{\mathrm{g}, \beta}$ and $T_{\mathrm{g}, \alpha}$, as determined using a linear regression methodology outlined previously [24] and shown in Table 8. It is worth noting that the values of $T_{\mathrm{g}, \alpha}$, as determined from the $\mathrm{THz}$ TDS experiments for F2 and F3, are in reasonable agreement with our own calorimetric measurements (Table 8), $T_{\mathrm{g}, \mathrm{mDSC}}$, as well as the values reported in the literature for these materials [28, 29, 30]. Notably, the value of the excipient mixture for F3 exhibits a relatively lower $T_{\mathrm{g}, \mathrm{mDSC}}$ value compared to $T_{\mathrm{g}, \alpha}$ observed by THz-TDS. 


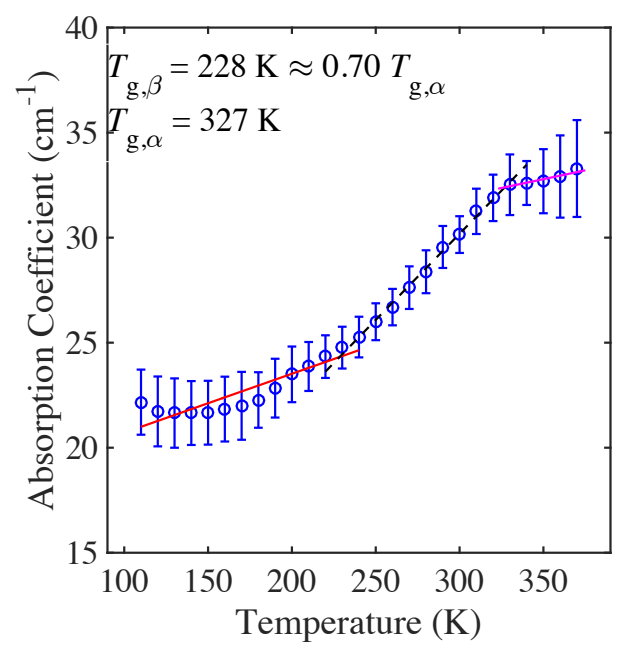

(a)

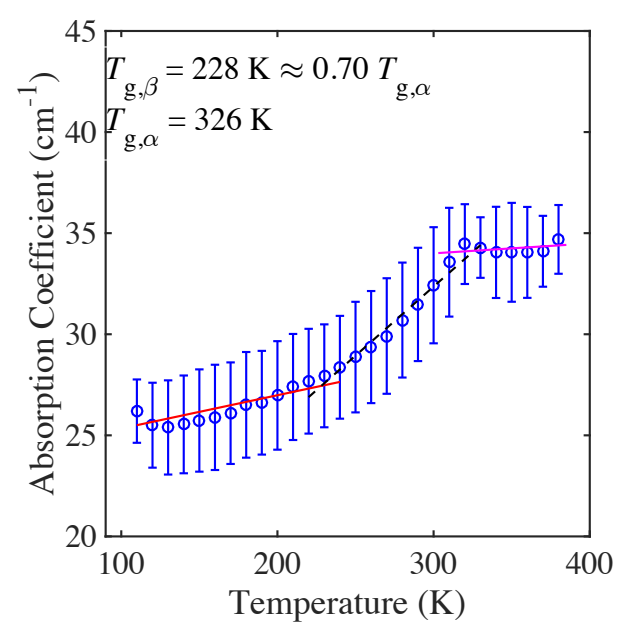

(c)

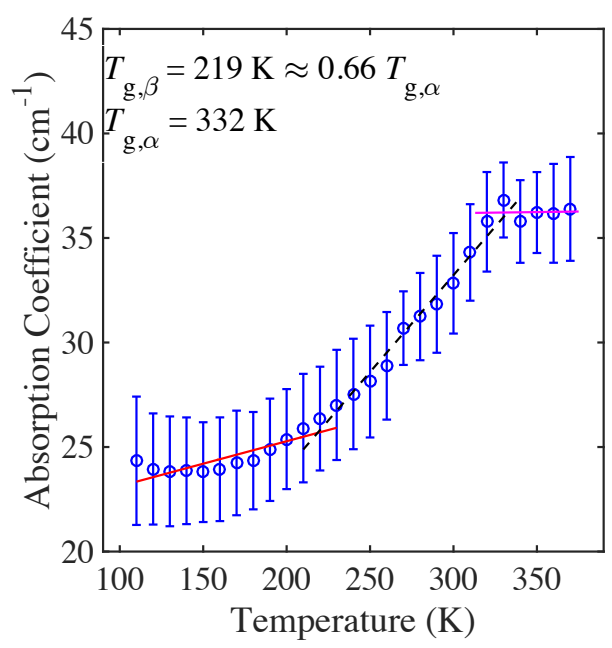

(b)

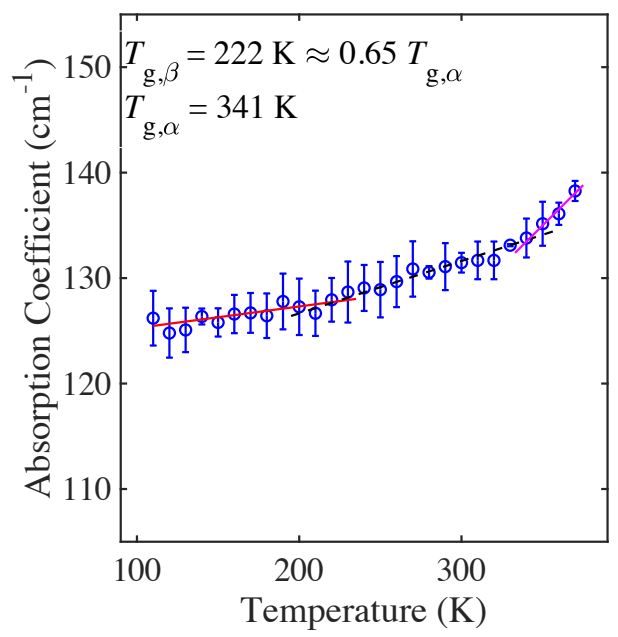

(d)

Figure 1: Mean terahertz absorption coefficient as a function of temperature at $1 \mathrm{THz}$ for formulations F1 (Figure 1a), F2 (Figure 1b), F3 (Figure 1c) and the spray-dried excipient mixture of F3, i.e. F3_Exc_SD (Figure 1d), respectively. The three lines show the linear fits for the three different regions. Error bars represent the standard deviation for $n=3$ samples. 
Table 8: Gradient, $m$, of the linear fit $(y=m x+c)$ for the respective temperature regions as outlined in Section 2.5.2 as well as the respective glass transition temperatures determined based on the terahertz analysis and mDSC. For all samples three regions were identified using the data analysis routine.

\begin{tabular}{|c|c|c|c|c|c|c|c|c|c|c|}
\hline \multirow[t]{2}{*}{ Formulation } & \multicolumn{2}{|c|}{$\begin{array}{c}m, \text { Region } 1 \\
\mathrm{~cm}^{-1} \mathrm{~K}^{-1}\end{array}$} & \multicolumn{2}{|c|}{$\begin{array}{c}m, \text { Region } 2 \\
\mathrm{~cm}^{-1} \mathrm{~K}^{-1}\end{array}$} & \multicolumn{2}{|c|}{$\begin{array}{c}m, \text { Region } 3 \\
\mathrm{~cm}^{-1} \mathrm{~K}^{-1}\end{array}$} & \multirow{2}{*}{$\begin{array}{c}T_{\mathrm{g}, \beta} \\
\mathrm{K} \\
n=3\end{array}$} & \multirow{2}{*}{$\begin{array}{c}T_{\mathrm{g}, \alpha} \\
\mathrm{K} \\
n=3\end{array}$} & \multirow{2}{*}{\multicolumn{2}{|c|}{ 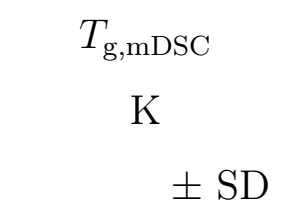 }} \\
\hline & $n=3$ & $\pm \mathrm{SD}$ & $n=3$ & $\pm \mathrm{SD}$ & $n=3$ & $\pm \mathrm{SD}$ & & & & \\
\hline $\mathrm{F} 1$ & 0.028 & 0.0038 & 0.082 & 0.0019 & 0.017 & 0.0036 & 228 & 327 & \multicolumn{2}{|c|}{$\mathrm{N} / \mathrm{A}^{\mathrm{a}}$} \\
\hline $\mathrm{F} 2$ & 0.022 & 0.0042 & 0.093 & 0.0045 & 0.0010 & 0.012 & 219 & 332 & 328.09 & $\mathrm{~N} / \mathrm{A}^{\mathrm{b}}$ \\
\hline F3 & 0.017 & 0.0032 & 0.068 & 0.0042 & 0.0049 & 0.0047 & 228 & 326 & 316.84 & $\mathrm{~N} / \mathrm{A}^{\mathrm{b}}$ \\
\hline F3_Exc_S & 10.021 & 0.0077 & 0.050 & 0.0016 & 0.15 & 0.022 & 222 & 341 & 312.78 & $1.56^{\mathrm{c}}$ \\
\hline
\end{tabular}

\footnotetext{
${ }^{\text {a }} T_{\mathrm{g}, \mathrm{mDSC}}$ could not be determined

${ }^{\mathrm{b}} n=1$ due to sample quantity restrictions

${ }^{\mathrm{c}} n=3$
} 
It is important to note that while the fit in Region 1 (see Figure 1) deviates from linearity, in the absence of clear physical mechanistic insight we cannot conclusively determine whether a non-linear model would be better suited. Given the qualitative trend of the data we have briefly explored, strictly in statistical terms, the suitability of a power law as a simple and commonly used model with a limited number of fitting parameters. To this end we have fit the data in Region 1 with a power law of the form $y=a x^{b}+c$ and compared the sum of squared errors (SSE) and root mean square error (RMSE) between the linear fit and the power law fit for each individual region as well as the overall data set (see ESI). We found there to be no significant difference in the magnitude of errors which would favour the power law fit over the linear fit in statistical terms. In our previous work on lyophilised formulations, which were of similar composition to F1-F3 that were investigated in this study[31], we did not observe a deviation from the linear change in absorption below $T_{\mathrm{g}, \beta}$. In the absence of clear compelling mechanistic insight into the observed behaviour we have chosen to retain a linear fit for temperatures $T<T_{\mathrm{g}, \beta}$ in all formulations but note that our observation should be explored in depth to explain the experimental behaviour. A short discussion on this topic can be found in the ESI.

Maintaining the native conformational state of BSA is critical in preserving its activity. However, this is challenging, as the spray-drying process exposes BSA to various stresses including heat, air-liquid interfacial stress, dehydration, and shearing of BSA during atomisation. Likewise, lyophilisation exposes BSA to a range of stresses, including freezing, dehydration and solid-liquid interfacial stresses [32]. In contrast to the lyophilisation process, 
for which the solid product has a moisture content of $1-3 \%$, spray-dried products have moisture contents ranging from $4-10 \%$ [2]. An increase in moisture content of the solid protein formulation has been associated with a decrease in the chemical stability of the protein [2], and has also been linked with increased molecular mobility [7, 33]. Using the THz-TDS method, we can track the changes in molecular mobility of each system by observing the changes in the absorption coefficient with temperature for each of the different formulations.

Changes in flexibility and molecular mobility associated with the conformational stability of BSA, both during drying and when subjected to heating during the THz-TDS experiments, are strongly influenced by the presence of trehalose, L-arginine $\mathrm{HCl}$, and polysorbate 20 as evident from the THz-TDS data. As water is removed during spray-drying, excipients like trehalose can form a considerably more rigid matrix to which the protein subsequently couples instead of water. This happens largely by means of hydrogen bonds but also other, weaker dispersion interaction, either directly to the matrix forming excipient or by means of a small intermediate molecule capable of forming hydrogen bonds, such as water, glycerol, or other excipients. The presence of the matrix forming excipient thus limits the protein mobility while ensuring a hydrogen bond rich environment to stabilise the protein structure [5, 33]. During spray-drying of formulation F1, the water molecules that stabilise the protein structure are successively replaced by trehalose molecules throughout the drying process to form an interconnected trehalose matrix [34, 35], where trehalose forms a strong hydrogen-bonded network with BSA. For F2, the positively charged amino acid L-arginine can bind to the negatively charged 
surfaces of BSA $[36,37]$ via electrostatic interactions in addition to the BSAtrehalose interactions. Furthermore, the guanidinium group of L-arginine can form salt bridges with acidic residues of the BSA molecules, which would introduce steric hindrance [38] and reduce the free volume of F2. For F3, polysorbate can additionally protect BSA from surface adsorption [6] during spray-drying.

We have previously shown that the different molecular motions of each system with changes in temperature can be tracked using THz-TDS [24]. The slope, $m$ (shown in Table 8), corresponds to the changes in absorption coefficient at $1 \mathrm{THz}$ with temperature, and can be directly linked to the molecular mobility of the system. For the spray-dried formulations (Figure 1a, Figure $1 \mathrm{~b}$, and Figure 1c), the linear gradient in the region of $T<T_{\mathrm{g}, \beta}$ is lower compared to that of the $T_{\mathrm{g}, \beta}<T<T_{\mathrm{g}, \alpha}$ region, indicating reduced or low molecular mobility at low temperatures [24]. In the region above $T_{\mathrm{g}, \alpha}$, the gradient decreases relative to the region of $T_{\mathrm{g}, \beta}<T<T_{\mathrm{g}, \alpha}$, and remains at a plateau at high temperatures. This indicates that at high temperatures the molecular mobility of the system becomes restricted, or confined, for the investigated spray-dried formulations. Notably, we have previously observed this behaviour for lyophilised BSA and mAb formulations [31]. We found that such confinement is not universal and only was observed for some of the lyophilised samples. We hypothesise that at $T<T_{\mathrm{g}, \beta}$, the conformations of BSA molecules are trapped in the local energy minima of their potential energy surface. For the BSA/excipient matrix, the BSA protein molecules can be envisioned as comparatively rigid clusters of hydrogen-bonded domains. Molecular motions are limited to localised thermal vibrations due to 
$k T$ that do not have sufficient amplitude to exceed the local barriers that restrict the molecular confinement. At $T>T_{\mathrm{g}, \beta}$, sufficient thermal energy and free volume are available to overcome these barriers [24,39] allowing for larger domains of the protein to become flexible and able to move with respect to each other but without losing the tertiary structure. This flexibility results in molecular mobility of dipoles that couple to the terahertz radiation and hence result in the experimentally observed increase in absorption with temperature. As the number of dipoles does not increase, the change in absorption is directly proportional to the increase in molecular mobility. At $T>T_{\mathrm{g}, \alpha}$, the protein molecules in F1-F3 become trapped by their surrounding matrix and the system appears to be confined in an energy minimum. In this region, the molecular flexibility, and with it the number of accessible conformational states is reduced, and thus, we do not observe any significant change in absorption for any of the BSA containing formulations.

When comparing the behaviour of the different formulations, F2 exhibits the lowest $T_{\mathrm{g}, \beta}$ value $\left(T_{\mathrm{g}, \beta}=219 \mathrm{~K}\right)$. This suggests that the molecules in F2 have to overcome a lower energy barrier for the onset of local mobility, compared to the molecules in F1 and F3 $\left(T_{\mathrm{g}, \beta}=228 \mathrm{~K}\right)$. Furthermore, the absolute value of the gradient between $T_{\mathrm{g}, \beta}$ and $T_{\mathrm{g}, \alpha}$ is approximately 5 times higher for F2 compared to F1 and F3 (Table 8). Previous work has shown that an increase in the strength of the hydrogen bonding network of a system, results in a corresponding decrease in the molecular mobility of the system [40]. Notably, for F1 we observe the most pronounced apparent deviation from a linear change in absorption with temperature for $T<T_{\mathrm{g}, \beta}$. Given this potential deviation from linear behaviour we have explored the use of a power 
law instead of a linear equation to fit the data in Region 1 for all formulations (see supporting information). One explanation of non-linear behaviour could be due to the increased concentration of water that is present in the spraydried samples when compared to lyophilised samples of similar composition that we studied previously but where no evidence of non-linearity at $T<$ $T_{\mathrm{g}, \beta}$ was found [31]. Specifically, the presence of a larger number of water molecules, which are relatively tightly bound to the protein molecules, could enhance the flexibility of the protein, similar to a plasticiser in a polymer, by further increasing the degrees of freedom of the system. In turn, the potential energy barriers that restrict motion at low temperatures would be reduced due to the relatively higher mobility of the strong dipoles of the water molecules in close vicinity to the protein molecules. Such behaviour would result in a wider distribution of low barrier states that confine the onset of initial protein motion and hence a more gradual onset of motion upon heating would be intuitive compared to a formulation where there is less water present. Notably, following the error analysis routine comparing the linear fit versus the power law fit in Region 1 (see ESI), we cannot definitively conclude that a power law fit is the best fit. While a non-linear change in absorption below $T<T_{\mathrm{g}, \beta}$ could be due to a phase transition in water taking place in this temperature region $[21,22,41,42]$ for the spraydried formulations, such assignment is tentative and further work would be required to challenge this hypothesis (see ESI). Hence, we have used a linear fit for Region 1 of F1-F3 in line with the methodology we have previously implemented on lyophilised formulations which exhibited no deviation from linearity [31] and are of similar composition to F1-F3. 
In order to investigate the role of BSA versus the excipients alone, we measured the change in terahertz absorption for a blank excipient matrix (Figure 1d). The measurement of this spray-dried excipient mixture indicates two critical observations when compared to those of F1-F3: (i) the excipient mixture exhibits the highest value of $T_{\mathrm{g}, \alpha}$, and (ii) the absorption coefficient of the excipient mixture continuously increases with temperature, with no indication of a plateau. The comparison of F3 (Figure 1c) to a spray-dried mixture of its excipients alone (Figure 1d), shows that a plateau at $T>T_{\mathrm{g}, \alpha}$ (i.e. confinement of molecular flexibility) depends on the presence of BSA. The presence of BSA molecules facilitates the formation of a stronger hydrogen bonding network at temperatures above $T_{\mathrm{g}, \alpha}$, and in the case of F3 this leads to a reduction in $T_{\mathrm{g}, \alpha}$ and a plateau in molecular mobility compared to the excipient mix alone. In the absence of BSA, the $T_{\mathrm{g}, \alpha}$ and molecular mobility increase for the weaker hydrogen bonded network, which can be observed in the THz-TDS data for excipient mixture which does not include BSA (Figure 1d) and is in line with our previous measurements of small organic molecular materials as well as polymers $[8,24]$. In the present study we observe evidence that the presence of proteins can result in a stronger hydrogen bonding network and even result in confinement of the molecular motions such as that they become trapped. It is important to highlight that this is not happening as a matter of principle for all formulations where BSA is present, but that it requires a specific interplay between the molecular species present in the formulation. Given the process of spray-drying, it is likely that each system includes an ensemble of distinct initial conformations, as even native BSA samples typically already contain some level of oligomers [43]; therefore, 
the point at which the different molecules and molecular species will become mobile enough and subsequently trapped above $T_{\mathrm{g}, \alpha}$ will differ. Moreover, aside from initial conformations of the protein molecules, the interactions between the BSA molecules and the excipients or residual solvents present in the formulation will affect the values of the transition temperatures. Notably, the limiting factors are the number of accessible conformational states available, free volume, and the thermal energy input into the system.

\subsection{Thermal Gradient FTIR Spectroscopy}

FTIR measurements were conducted in order to probe potential changes in the secondary protein structure during heating in order to provide context to the THz-TDS measurements. Specifically the amide I and amide II bands were evaluated. Peak shifts were first assessed visually on the overlaid spectra (see Figure 2), followed by evaluation of the peaks identified using the second derivative method described in Section 2.6. Applying the second derivative of the spectra did not reveal any additional peaks or peak shifts. Both amide bands were evaluated since the $\mathrm{H}-\mathrm{O}-\mathrm{H}$ bending vibration of water absorbs strongly around $1650 \mathrm{~cm}^{-1}$, thus overlapping with the amide I C=O stretch vibration $\left(1700-1600 \mathrm{~cm}^{-1}\right)$. Therefore, even though amide I is generally considered to be the more sensitive band with regard to changes in the secondary structure, amide II was also included in the assessment as evaporation of residual water (see Table 3) from the spray-dried samples during heating might influence amide I band characteristics [27]. Despite the overall absorption intensity increasing with temperature, no significant changes were observed in the relative peak intensities examined. Spectra recorded for F1 and F2 can be found in the supporting information. 
No clear peak shifts could be observed in the amide I and amide II band for any of the formulations. At first sight, the lack of observable changes in the secondary structure by FTIR during heating appears to be in contrast with the increase in molecular motions that was observed using THz-TDS. However, differences in the energy levels that are probed with both techniques respectively, should be taken into further consideration. To put the measurements into context, it is helpful to consider the photon energy of the the amide I and amide II bands, as well as the frequency of $1 \mathrm{THz}$, that was used for THz-TDS analysis: There is more than a factor of fifty difference in the energy levels probed using FTIR spectroscopy $\left(\approx 3^{-20} \mathrm{~J}\right)$ and THzTDS $\left(\approx 7^{-22} \mathrm{~J}\right)$. The bond strength sampled in vibrational spectroscopy is proportional to aforementioned photon energy and inversely proportional to the mass of the vibrating oscillator. It is therefore obvious that sensitivity to subtle conformational variations is far higher at terahertz frequencies than at those probed using FTIR, as the subtle energies dominating intermolecular interactions such as hydrogen bonding, van der Waals and other dispersive forces can be directly probed at terahertz frequencies.

In summary, FTIR analysis did not reveal any changes in secondary structure for the formulations examined, with the sensitivity of FTIR being inherently insufficient to reliably resolve subtle changes in the flexibility of the system.

\section{Conclusions}

We have examined three unique spray-dried formulations, each containing BSA. By performing temperature variable THz-TDS experiments we stud- 


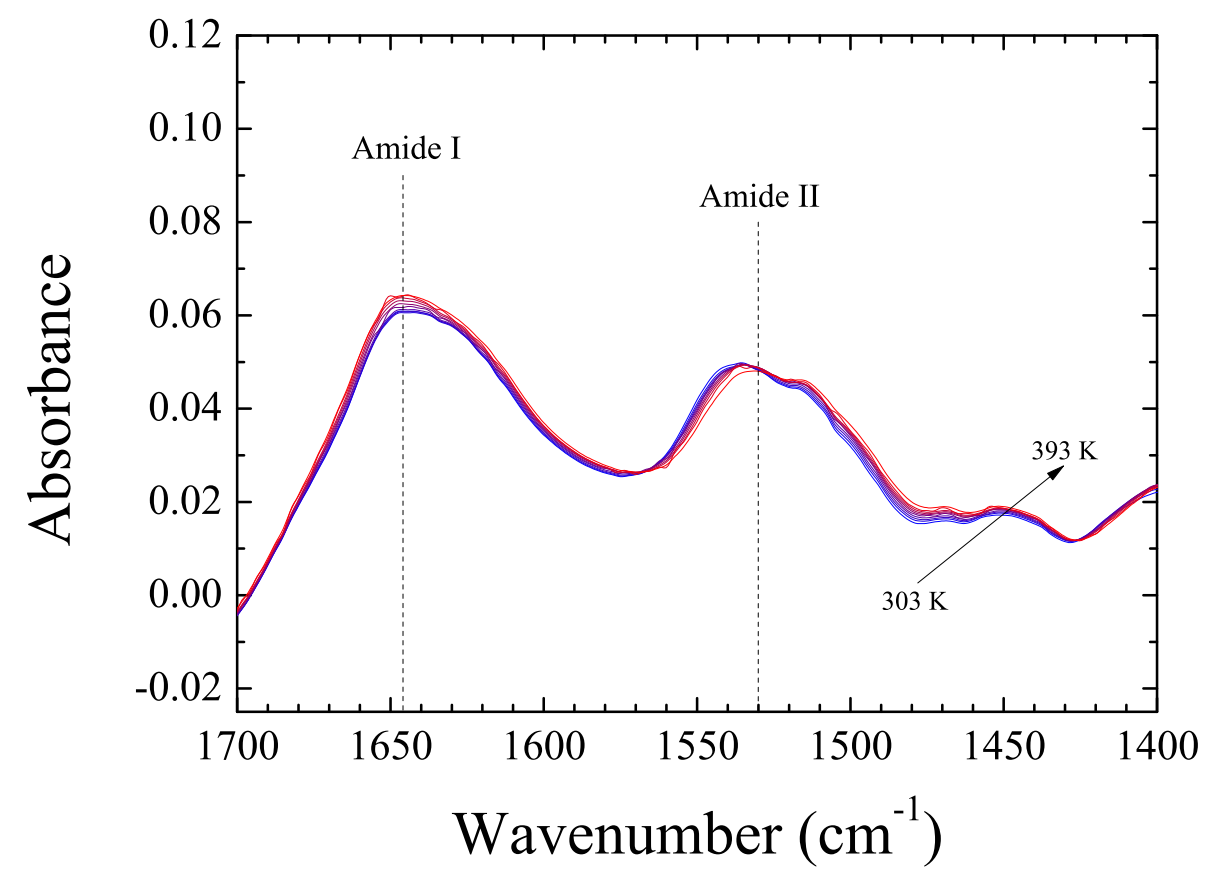

Figure 2: Overlay of FTIR spectra collected for F3 at different temperatures. Colour coding indicates the temperature gradient ranging from low (blue) to high (red). Dashed vertical lines at $1646 \mathrm{~cm}^{-1}$ and $1533 \mathrm{~cm}^{-1}$ serve as reference lines for the amide I (1700 $\left.\mathrm{cm}^{-1}-1600 \mathrm{~cm}^{-1}\right)$ and amide II absorption band $\left(1580 \mathrm{~cm}^{-1}-1520 \mathrm{~cm}^{-1}\right)$, respectively. 
ied the changes in structural dynamics that these formulations can undergo. Furthermore, we used thermal gradient FTIR to evaluate the conformational stability of the protein in the solid state during heating. We have shown that the $T_{\mathrm{g}, \alpha}$ values found based on the THz-TDS data, are relatively consistent with the $T_{\mathrm{g}}$ results determined by $\mathrm{mDSC}$ for each material. We have found evidence for vibrational confinement in all BSA containing formulations at temperatures above $T_{\mathrm{g}, \alpha}$ following spray-drying and have shown that the absorption coefficient reaches a plateau at high temperatures for all the BSA formulations. This observation is in contrast to previously studied lyophilised formulations, where we observed such confinement only in a select number of cases [31]. The results presented in this work indicate an effect of molecular mobility confinement above $T_{\mathrm{g}, \alpha}$, and a forthcoming publication by the authors will investigate the significance of our observations for the solid state stability of protein formulations. Our findings are likely correlated to the (protein) stability of the formulations, but further research involving stability analysis is required before any conclusions can be drawn regarding this subject. The presented work put forward a physical hypothesis connecting the measured experimental data to temperature induced changes in the conformational state of BSA and the potential influence of the excipients on these states. The work thus provides a framework for better understanding the dynamics of complex formulations and demonstrates that THz-TDS is an effective method for measuring the molecular dynamics and temperaturedependant behaviour of spray-dried solid-state formulations. 


\section{Acknowledgements}

UCB Pharma are gratefully acknowledged for providing the equipment, materials and funding to make this study possible.

T.A.S. and J.A.Z. acknowledge funding from AstraZeneca UK Limited/MedImmune Limited and the UK Engineering and Physical Sciences Research Council (EP/N022769/1). T.A.S. would like to thank the AJA-Karten Trust and the AIA-Kenneth Lindsay Trust for their financial support.

[1] A. Sosnik, K. P. Seremeta, Advantages and challenges of the spraydrying technology for the production of pure drug particles and drugloaded polymeric carriers, Adv. Colloid Interface Sci. 223 (2015) 40-54. doi:10.1016/j.cis.2015.05.003.

[2] M. Ameri, Y.-F. Maa, Spray drying of biopharmaceuticals: Stability and process considerations, Dry. Technol. 24 (6) (2006) 763-768. doi: $10.1080 / 03602550600685275$.

[3] R. Pentewar, S. Somwanshi, B. Sugave, Spray drying: A review on single step rapid drying technique, Res. J. Pharm. Biol. Chem. Sci. 5 (2014) $1502-1514$.

[4] N. Soltanizadeh, L. Mirmoghtadaie, F. Nejati, L. I. Najafabadi, M. K. Heshmati, M. Jafari, Solid-state protein-carbohydrate interactions and their application in the food industry, Comp. Rev. Food Sci. F. 13 (5) (2014) 860-870. doi:10.1111/1541-4337.12089.

[5] K. Rajagopal, J. Wood, B. Tran, T. W. Patapoff, T. Nivaggioli, Trehalose limits BSA aggregation in spray-dried formulations at high tem- 
peratures: Implications in preparing polymer implants for long-term protein delivery, J. Pharm. Sci. 102 (8) (2013) 2655-2666. doi:10. 1002/jps. 23634.

[6] T. Arakawa, Y. Kita, Protection of bovine serum albumin from aggregation by Tween 80, J. Pharm. Sci. 89 (5) (2000) 646-651. doi:10.1002/ (SICI) 1520-6017 (200005) 89: 5<646: : AID- JPS10>3 . 0 . C0 ; 2- J.

[7] M. Pikal, S. Shah, M. L. Roy, R. Putman, The secondary drying stage of freeze drying: Drying kinetics as a function of temperature and chamber pressure, Int. J. Pharm. 60 (1990) 203-207. doi:10.1016/ 0378-5173(90)90074-E.

[8] J. Sibik, J. A. Zeitler, Direct measurement of molecular mobility and crystallisation of amorphous pharmaceuticals using terahertz spectroscopy, Adv. Drug Deliv. Rev. 100 (2016) 147-157. doi:10.1016/j. addr.2015.12.021.

[9] H.-B. Yu, W.-H. Wang, K. Samwer, The $\beta$ relaxation in metallic glasses: An overview, Mater. Today 16 (5) (2013) 183-191. doi:10.1016/j. mattod.2013.05.002.

[10] A. Alegria, J. Colmenero, Dielectric relaxation of polymers: Segmental dynamics under structural constraints, Soft Matter 12 (37) (2016) 77097725. doi :10.1039/C6SM01298A.

[11] S. Cerveny, R. Bergman, G. A. Schwartz, P. Jacobsson, Dielectric $\alpha$ and $\beta$-relaxations in uncured styrene butadiene rubber, Macromolecules 35 (11) (2002) 4337-4342. doi : 10.1021/ma010990o. 
[12] A. K. Roy, P. T. Inglefield, Solid state NMR studies of local motions in polymers, Prog. Nucl. Magn. Reson. Spectrosc. 22 (6) (1990) 569-603. doi:10.1016/0079-6565(90)80009-7.

[13] M. D. Barnes, K. Fukui, K. Kaji, T. Kanaya, D. W. Noid, J. U. Otaigbe, V. N. Pokrovskii, B. G. Sumpter, Advances in Polymer Science Polymer Physics and Engineering, 1st Edition, Springer, Berlin Heidelberg, Germany, 2001.

[14] K. L. Ngai, Relation between some secondary relaxations and the $\alpha$ relaxations in glass forming materials according to the coupling model., J. Chem. Phys. 109 (16) (1998) 6982. doi:10.1063/1.477334.

[15] J. Sibik, S. R. Elliott, J. A. Zeitler, Thermal decoupling of molecularrelaxation processes from the vibrational density of states at terahertz frequencies in supercooled hydrogen-bonded liquids, J. Phys. Chem. Lett. 5 (11) (2014) 1968-1972. doi:10.1021/jz5007302.

[16] S. Capaccioli, K. L. Ngai, M. S. Thayyil, D. Prevosto, Coupling of caged molecule dynamics to JG $\beta$-relaxation: I, J. Phys. Chem. B 119 (28) (2015) 8800-8808. doi:10.1021/acs .jpcb.5b04408.

[17] G. P. Johari, M. Goldstein, Viscous liquids and the glass transition. II. secondary relaxations in glasses of rigid molecules, J. Chem. Phys. 53 (6) (1970) 2372-2388. doi:10.1063/1.1674335.

[18] G. Williams, D. C. Watts, Molecular motion in the glassy state. The effect of temperature and pressure on the dielectric $\beta$ relaxation of 
polyvinyl chloride, Trans. Faraday Soc. 67 (0) (1971) 1971-1979. doi: 10.1039/TF9716701971.

[19] M. Goldstein, Viscous Liquids and the Glass Transition: A Potential Energy Barrier Picture, J. Chem. Phys. 51 (9) (1969) 3728. doi:10. 1063/1.1672587.

[20] M. Batens, J. Massant, B. Teodorescu, G. V. den Mooter, Formulating monoclonal antibodies as powders for reconstitution at high concentration using spray drying: Models and pitfalls, Eur. J. Pharm. Biopharm. 127 (2018) 407-422. doi:10.1016/j.ejpb.2018.02.002.

[21] M. M. Koza, B. Geil, H. Schober, F. Natali, Absence of molecular mobility on nano-second time scales in amorphous ice phases, Phys. Chem. Chem. Phys. 7 (2005) 1423-1431. doi:10.1039/B414382E.

[22] C. A. Angell, Insights into phases of liquid water from study of its unusual glass-forming properties, Science 319 (5863) (2008) 582587. arXiv:https://science.sciencemag.org/content/319/5863/ 582.full.pdf, doi:10.1126/science.1131939.

[23] M. Mizuno, M. J. Pikal, Is the pre-tg dsc endotherm observed with solid state proteins associated with the protein internal dynamics? investigation of bovine serum albumin by solid state hydrogen/deuterium exchange, Eur. J. Pharm. Biopharm. 85 (2) (2013) 170-176. doi: 10.1016/j.ejpb.2013.04.019.

[24] T. A. Shmool, J. A. Zeitler, Insights into the structural dynamics of poly 
lactic-co-glycolic acid at terahertz frequencies, Polym. Chem. 10 (2019) 351-361. doi:10.1039/C8PY01210E.

[25] L. Duvillaret, F. Garet, J. L. Coutaz, A reliable method for extraction of material parameters in terahertz time-domain spectroscopy, IEEE J. Sel. Top. Quantum Electron. 2 (3) (1996) 739-746. doi:10.1109/2944. 571775 .

[26] J. Sibik, J. A. Zeitler, Terahertz response of organic amorphous systems: Experimental concerns and perspectives, Philos. Mag. 96 (7-9) (2015) 842-853. doi:10.1080/14786435.2015.1111528.

[27] M. van de Weert, L. Jørgensen, Infrared Spectroscopy to Characterize Protein Aggregates, Wiley: Hoboken, New Jersey, United States, 2012, Ch. 10, pp. 227-248. arXiv:https:// onlinelibrary .wiley .com/doi/pdf/10.1002/9781118150573.ch10, doi:10.1002/9781118150573.ch10.

[28] S. P. Duddu, P. R. Dal Monte, Effect of glass transition temperature on the stability of lyophilized formulations containing a chimeric therapeutic monoclonal antibody, Pharm. Res. 14 (5) (1997) 591-595. doi:110.1023/A.1012144810067.

[29] P. Srirangsan, K. Kawai, N. Hamada-Sato, M. Watanabe, T. Suzuki, Improvement in the remaining activity of freeze-dried xanthine oxidase with the addition of a disaccharide-polymer mixture, Food Chem. 119 (1) (2010) 209-213. doi:10.1016/j.foodchem.2009.06.016. 
[30] B. Wang, S. Tchessalov, M. T. Cicerone, N. W. Warne, M. J. Pikal, Impact of sucrose level on storage stability of proteins in freeze-dried solids: II. Correlation of aggregation rate with protein structure and molecular mobility, J. Pharm. Sci. 98 (9) (2009) 3145-3166. doi:10. 1002/jps.21622.

[31] T. A. Shmool, P. J. Hooper, M. Leutzsch, A. D. Stephens, M. U. Gaimann, M. D. Mantle, G. S. Kaminski Schierle, C. F. van der Walle, J. A. Zeitler, Observation of high temperature macromolecular confinement in lyophilised protein formulations using terahertz spectroscopy, ChemRxiv. (2019) 8100164doi:10.26434/chemrxiv.8100164.

[32] B. S. Moorthy, L. K. Iyer, E. M. Topp, Characterizing protein structure, dynamics and conformation in lyophilized solids, Curr. Pharm. Des. 21 (40) (2015) 5845-5853. doi:10.2174/1381612821666151008150735.

[33] M. T. Cicerone, J. F. Douglas, $\beta$-Relaxation governs protein stability in sugar-glass matrices, Soft Matter 8 (10) (2012) 2983-2991. doi: 10.1039/C2SM06979B.

[34] S. Sonavane, P. Chakrabarti, Cavities and atomic packing in protein structures and interfaces, PLoS Comput. Biol. 4 (9) (2008) e1000188. doi:10.1371/journal.pcbi.1000188.

[35] N. K. Jain, I. Roy, Effect of trehalose on protein structure, Protein Sci. 18 (1) (2009) 24-36. doi:10.1002/pro.3.

[36] V. A. Borzova, K. A. Markossian, S. Y. Kleymenov, B. I. Kurganov, A change in the aggregation pathway of bovine serum albumin in the 
presence of arginine and its derivatives, Sci. Rep. 7 (1) (2017) 3984. doi:10.1038/s41598-017-04409-x.

[37] D. Shukla, B. L. Trout, Interaction of arginine with proteins and the mechanism by which it inhibits aggregation, J. Phys. Chem. B 114 (42) (2010) 13426-13438. doi:10.1021/jp108399g.

[38] M. Reslan, Y. K. Demir, B. L. Trout, H.-K. Chan, V. Kayser, Lack of a synergistic effect of arginine-glutamic acid on the physical stability of spray-dried bovine serum albumin, Pharm. Dev. Technol. 22 (6) (2017) 785-791. doi:10.1080/10837450.2016.1185116.

[39] M. T. Ruggiero, M. Krynski, E. O. Kissi, J. Sibik, D. Markl, N. Y. Tan, D. Arslanov, W. van der Zande, B. Redlich, T. M. Korter, H. Grohganz, K. Löbmann, T. Rades, S. R. Elliott, J. A. Zeitler, The significance of the amorphous potential energy landscape for dictating glassy dynamics and driving solid-state crystallisation, Phys. Chem. Chem. Phys. 19 (44) (2017) 30039-30047. doi:10.1039/c7cp06664c.

[40] M. A. Mensink, H. W. Frijlink, K. van der Voort Maarschalk, W. L. J. Hinrichs, How sugars protect proteins in the solid state and during drying (review): Mechanisms of stabilization in relation to stress conditions, Eur. J. Pharm. Biopharm. 114 (Supplement C) (2017) 288-295. doi:10.1016/j.ejpb.2017.01.024.

[41] H. Goshima, K. M. Forney Stevens, M. Liu, K. K. Qian, M. Tyagi, M. T. Cicerone, M. J. Pikal, Addition of monovalent electrolytes to 
improve storage stability of freeze-dried protein formulations, J. Pharm. Sci. 105 (2) (2016) 530-541. doi:10.1016/j.xphs.2015.10.004.

[42] S. Loppolo, B. A. McGuire, M. A. Allodi, G. A. Blake, THz and mid-IR spectroscopy of interstellar ice analogs: Methyl and carboxylic acid groups, Faraday Discuss. 168 (2014) 461-484. doi:10.1039/ C3FD00154G.

[43] Y. Wei, C. Wang, B. Jiang, C. C. Sun, C. R. Middaugh, Developing biologics tablets: The effects of compression on the structure and stability of bovine serum albumin and lysozyme, Mol. Pharm. 16 (3) (2019) 1119-1131. arXiv:https://doi.org/10.1021/acs.molpharmaceut. 8b01118, doi:10.1021/acs.molpharmaceut.8b01118. 